\title{
A novel aldose-aldose oxidoreductase for co-production of D-xylonate and xylitol from D-xylose with Saccharomyces cerevisiae
}

\author{
Marilyn G. Wiebe ${ }^{1} \cdot$ Yvonne Nygård $^{1} \cdot$ Merja Oja $^{1}$ • Martina Andberg ${ }^{1}$. \\ Laura Ruohonen $^{1} \cdot$ Anu Koivula ${ }^{1} \cdot$ Merja Penttilä $^{1} \cdot$ Mervi Toivari $^{1}$
}

Received: 14 April 2015 /Revised: 7 July 2015 / Accepted: 21 July 2015 / Published online: 12 August 2015

(C) The Author(s) 2015. This article is published with open access at Springerlink.com

\begin{abstract}
An open reading frame CC1225 from the Caulobacter crescentus CB15 genome sequence belongs to the Gfo/Idh/MocA protein family and has $47 \%$ amino acid sequence identity with the glucose-fructose oxidoreductase from Zymomonas mobilis ( $\mathrm{Zm}$ GFOR). We expressed the ORF CC1225 in the yeast Saccharomyces cerevisiae and used a yeast strain expressing the gene coding for $Z m$ GFOR as a reference. Cell extracts of strains overexpressing CC1225 (renamed as Cc aaor) showed some $\mathrm{Zm}$ GFOR type of activity, producing D-gluconate and D-sorbitol when a mixture of $\mathrm{D}$-glucose and D-fructose was used as substrate. However, the activity in $C c$ aaor expressing strain was $>100$-fold lower compared to strains expressing $\mathrm{Zm}$ gfor. Interestingly, C. crescentus AAOR was clearly more efficient than the $Z m$ GFOR in converting in vitro a single sugar substrate D-xylose $(10 \mathrm{mM})$ to xylitol without an added cofactor, whereas this type of activity was very low with $Z m$ GFOR. Furthermore, when cultured in the presence of D-xylose, the S. cerevisiae strain expressing $C c$ aaor produced nearly equal concentrations of D-xylonate and xylitol (12.5 g D-xylonate $~^{-1}$ and $11.5 \mathrm{~g} \mathrm{D}$-xylitol $\mathrm{l}^{-1}$ from $26 \mathrm{~g} \mathrm{D}$-xylose $\mathrm{l}^{-1}$ ), whereas the control strain and strain expressing $Z m$ gfor produced only D-xylitol $\left(5 \mathrm{~g}^{-1}\right)$. Deletion of the gene encoding the major aldose reductase, Gre3p, did not affect xylitol production in the strain expressing $C c$ aaor, but decreased xylitol production in the strain expressing Zm gfor. In addition, expression of Cc aaor together with the D-xylonolactone lactonase encoding the gene $x y l C$ from $C$. crescentus slightly increased
\end{abstract}

Mervi Toivari

mervi.toivari@vtt.fi

1 VTT, Technical Research Centre of Finland Ltd., P.O. Box 1000, FI-02044 VTT, Espoo, Finland the final concentration and initial volumetric production rate of both D-xylonate and D-xylitol. These results suggest that C. crescentus AAOR is a novel type of oxidoreductase able to convert the single aldose substrate D-xylose to both its oxidized and reduced product.

Keywords D-Xylonic acid · Xylitol · D-Xylose · Glucose-fructose oxidoreductase · GFOR

\section{Introduction}

D-Xylose, the main component of hemicellulose, is the second most abundant sugar in nature after cellulose and starchderived D-glucose. D-Glucose is widely used in various applications and processes, whereas the use of the pentose sugar D-xylose is not yet fully established. New products and production processes are needed for efficient utilization of hemicellulose and D-xylose.

Various studies have shown possibilities for biotechnical conversion of D-xylose to ethanol, butanol, lactic acid, succinic acid, xylonic acid, xylitol, hydrogen, modified sugars (transglycosylation) or compounds derived from fatty acid metabolism (Dumon et al. 2012; Peng et al. 2012). These products are formed via metabolism by natural or engineered microbes, e.g. of species like Escherichia coli, Corynebacterium glutamicum, Zymomonas mobilis, Saccharomyces cerevisiae and Scheffersomyces stipitis. The first conversion step of D-xylose is mostly performed with either xylose isomerase (EC 5.3.1.5) converting D-xylose to D-xylulose, D-xylose reductase (EC 1.1.1.307 or EC 1.1.1.21) converting D-xylose to xylitol, or D-xylose dehydrogenase (EC 1.1.1.175 or EC 1.1.1.179) converting D-xylose to Dxylonate. These products can be used as such or converted further chemically or enzymatically. Xylitol and D-xylonate, 
the reduced and oxidized products of D-xylose, are the simplest conversion products with established or demonstrated applications (cf. D-xylulose). Xylitol is a widely used sweetener with, e.g. anti-caries properties. Xylitol is currently mainly produced by chemical reduction of D-xylose with a nickel catalyst, but D-xylose can also be reduced to xylitol using an aldose reductase enzyme in vitro or in vivo, e.g. in yeast (Nidetzky et al. 1996; Winkelhausen and Kuzmanova 1998; Hallborn et al. 1991). D-Xylose dehydrogenases or oxidases oxidize D-xylose to D-xylonate, which has applications, e.g. as a chelator or as a hydrogel modifier (Millner et al. 1994; Chun et al. 2006; Zamora et al. 2000). D-Xylonate is produced by various bacteria or as described more recently by recombinant microbes, such as E. coli, S. cerevisiae, Kluyveromyces lactis or Pichia kudriavzevii (Nygård et al. 2011; Toivari et al. 2012a, b; Toivari et al. 2013; Liu et al. 2012; Cao et al. 2013). The reactions for enzymatic oxidation or reduction of Dxylose have so far been performed with separate enzymes using $\mathrm{NADP}^{+} / \mathrm{NADPH}$ or $\mathrm{NAD}^{+} / \mathrm{NADH}$ (or alternatively $\mathrm{FAD}^{+} / \mathrm{FADH}$ or PQQ) as cofactors. Whole cell conversions are usually preferred over in vitro enzymatic conversions for redox-linked reactions because regeneration of the cofactor in large scale in vitro systems is expensive. However, it is also well known that introduction of a redox reaction affects the cellular cofactor pool and that optimal production may necessitate further engineering of redox metabolism, use of a cosubstrate or increased aeration. Some enzymes, such as the glucose-fructose oxidoreductase (EC 1.1.99.28), have a bound cofactor and can perform both oxidation and reduction reactions without interfering with the cellular redox balance (Piersma et al. 1997; Zachariou and Scopes 1986). Glucosefructose oxidoreductase activity generates two products, an acid and an alcohol, which could be applicable, e.g. in polymer synthesis.

The glucose-fructose oxidoreductase of $Z$. mobilis has been characterized and its crystal structure solved. It has remained the only characterized enzyme of this unique oxidoreductase type, in spite of its interesting properties with bound cofactor, a redox neutral reaction cycle and two products.

Our long-term interest in pentose sugar utilization has led to searches for novel D-xylose-converting enzymes, isomerases, reductases, and dehydrogenases. Some D-xylose dehydrogenases belong to the Gfo/Idh/MocA enzyme family, which also includes the glucose-fructose oxidoreductase of Z. mobilis. During studies on D-xylose dehydrogenases (Toivari et al. 2012a, b), we encountered an open reading frame (ORF) CC1225 from the fresh water bacterium C. crescentus (synonym Caulobacter vibrioides) automatically annotated as a glucose-fructose oxidoreductase or as a Dxylose dehydrogenase. To evaluate whether this ORF (CC1225) is involved in D-xylose conversion, we expressed it in $S$. cerevisiae, using the well-characterized GFOR of Z. mobilis as a reference enzyme.

\section{Materials and methods}

\section{Strain construction}

S. cerevisiae strain CEN.PK 113-17A (H2802; MAT $\alpha$, ura352 HIS3 leu2-3/112 TRP1 MAL2-8 SUC2) (Entian and Kötter 1998) was used as the parental strain for all genetic modifications. The strains used in the study are listed in Table 1.

The putative glucose-fructose oxidoreductase encoding gene from C. crescentus CB15 (CC1225, AAK23207.1, NCBI), hereafter called $C c$ aaor (gene) or $C c$ AAOR when referring to the enzyme), was obtained as a synthetic gene, codon optimized for S. cerevisiae, deposited sequence KR269738 (Gene Art, Germany). Similarly, the gene encoding the glucosefructose oxidoreductase of $Z$. mobilis (Q07982.2, NCBI), hereafter called Zm gfor (gene) or Zm GFOR (when referring to the enzyme), was obtained as a synthetic gene, codon optimized for S. cerevisiae, deposited sequence KR269739 (Genscript, New Jersey). The genes $C c$ aaor and $Z m$ gfor were ligated into the $B g l I I$ site, between the $P G K 1$ promoter and terminator, of YEplac195 + PGK1PT (B1181), generating plasmids B3353 and B3895, respectively. Both genes contained signal sequences for periplasmic translocation. The signal sequences were removed by amplifying the genes with primers CCTACTGAATTCAGATCTACAATGGCTCAACCAGGTAGAAAATTG and TTACCTGGATCCAGATCTTCACAAT TTAACAGTTCTA for $C c$ aaor and ATCGTAGAT CTAAGTTACTATGGCAACTTTACCTGCCGGTGC and TTGCAGAGATCTTCATTAGTAAC for Zm gfor using plasmids B3353 and B3895 as templates. The resulting $C c$ aaor and $Z m$ gfor fragments were cloned into the B1181 expression vector as described above, resulting in plasmids B4023 and B4022, for $C c$ aaor and $\mathrm{Zm}$ gfor, respectively.

Table 1 S. cerevisiae strains used in this study. w/o ss; without signal sequence

\begin{tabular}{|c|c|c|}
\hline Strain & Strain number & $\begin{array}{l}\text { Genotype or parent } \\
\text { strain + plasmid } \\
\text { (B-number) }\end{array}$ \\
\hline CEN.PK113-17A & $\mathrm{H} 2802$ & $\begin{array}{c}\text { MAT } \alpha, \text { ura3-52 HIS3 } \\
\text { leu2-3/112 TRP1 } \\
\text { MAL2-8 SUC2 }\end{array}$ \\
\hline control & VTT-C-15930 & $\mathrm{H} 2802+\mathrm{B} 1181$ \\
\hline Cc aaor & VTT-C-15928 & $\mathrm{H} 2802+\mathrm{B} 3353$ \\
\hline Cc aaor xylC & VTT-C-15929 & VTT-C-15928 + B3574 \\
\hline Zm gfor & VTT-C-15933 & $\mathrm{H} 2802+\mathrm{B} 3895$ \\
\hline Cc aaor w/o ss & VTT-C-15935 & $\mathrm{H} 2802+\mathrm{B} 4023$ \\
\hline Zm gfor w/o ss & VTT-C-15934 & $\mathrm{H} 2802+\mathrm{B} 4022$ \\
\hline$\triangle G R E 3$ & VTT-C-15927 & $\mathrm{H} 2802 \triangle G R E 3$ \\
\hline$\triangle G R E 3$ control & VTT-C-15931 & $\mathrm{H} 2802 \triangle G R E 3+\mathrm{B} 1181$ \\
\hline Cc aaor $\triangle G R E 3$ & VTT-C-15932 & $\mathrm{H} 2802 \triangle G R E 3+\mathrm{B} 3353$ \\
\hline
\end{tabular}


Plasmids B3353, B3895, B4023 and B4022 were introduced into $S$. cerevisiae strain $\mathrm{H} 2802$ to generate strains VTT-C-15928, VTT-C-15933, VTT-C-15935 and VTT-C15934, respectively (Table 1 ). A control strain was created by introducing plasmid B1181 to $S$. cerevisiae CEN.PK 11317A (H2802), resulting in strain VTT-C-15930. Plasmid B3353 was also introduced into the Gre3p-deficient strain VTT-C-15927 (H3613, Toivari et al. 2010), resulting in strain VTT-C-15932. A control strain was created by introducing plasmid B1181 to Gre3p-deficient strain VTT-C-15927, resulting in strain VTT-C-15931. Plasmid B3574 expressing xylonolactone lactonase encoding gene $x y l C$ from C. crescentus (Toivari et al. 2012a, b) was transformed to strain VTT-C-15928 resulting in strain VTT-C-15929.

\section{Media, culture conditions and measurement of biomass}

Yeast strains were cultured in $50 \mathrm{ml}$ of modified synthetic complete medium lacking uracil (SC-ura, modified from Sherman 1983) in 250-ml Erlenmeyer flasks, at $250 \mathrm{rpm}$, $30^{\circ} \mathrm{C}$. D-Glucose was used as a carbon source and D-xylose was added in concentrations indicated in the text. Calcium carbonate $\left(\mathrm{CaCO}_{3}, 1 \% \mathrm{w} / \mathrm{v}\right)$ was used to buffer the medium in flask cultures. In bioreactors, yeasts were grown in $500 \mathrm{ml}$ medium (SC-ura) in Multifors bioreactors (max. working volume $500 \mathrm{ml}$, Infors HT, Switzerland) at $\mathrm{pH} \mathrm{5.5,} 30^{\circ} \mathrm{C}, 1$ volume air [volume culture] ${ }^{-1} \mathrm{~min}^{-1}$ (vvm) and $500 \mathrm{rpm}$ agitation with 2 marine impellors (Toivari et al. 2010). The $\mathrm{pH}$ was maintained constant by addition of $2 \mathrm{M} \mathrm{NaOH}$ or $1 \mathrm{M}$ $\mathrm{H}_{2} \mathrm{PO}_{4}$. Clerol antifoaming agent (Cognis, France, 0.08$0.10 \mu \mathrm{l}^{-1}$ ) was added to prevent foam formation.

Biomass was measured as optical density (OD) at $600 \mathrm{~nm}$ $\left(\mathrm{OD}_{600}\right)$ and/or as dry weight. For cultures containing $\mathrm{CaCO}_{3}$, OD was determined by diluting the sample 6-fold with $4 \mathrm{~N}$ $\mathrm{HCl}$ to solubilize $\mathrm{CaCO}_{3}$. Subsequent dilutions, if needed, were made in water. For dry weight, samples were collected in 2-ml pre-dried, pre-weighed microcentrifuge tubes, washed twice with equal volume distilled water and dried at $100^{\circ} \mathrm{C}$.

\section{Bioinformatic analysis}

The similarity relationship of the $C c$ AAOR protein coding sequence to the Gfo/Idh/MocA family, especially to D-xylose and L-arabinose dehydrogenases, was mapped from a protein BLAST search (http://blast.ncbi.nlm.nih.gov). Cc AAOR sequence together with $\mathrm{Zm}$ GFOR, and the known D-xylose or L-arabinose, or putative L-arabinose dehydrogenase sequences of the Gfo/Idh/MocA family were used as query sequences in a BLAST search against the UniProt database (http://www.uniprot.org/). The query protein sequences were as follows: C. crescentus AAOR (Q9A8X3), T. reesei Dxylose DH (A8BT09), H. marismortui xylose DH (Q5UY95), Z. mobilis GFOR (Q07982), A. brasiliense L- arabinose DH (Q53TZ2), R. leguminosarum L-arabinose DH (B5ZWY9), Bradyrhizobium L-arabinose DH (A5EDS7), P. fluorescens L-arabinose DH (P11886), B. thailandesis DH (Q2T4S6), B. japonicum DH (Q89QC3), R. meliloti galactose DH (Q92QY5), M. fascicularis trans1,2-dihydrobenzene-1,2-diol DH (Q9TQS6) and pig liver trans-1,2-dihydrobenzene-1,2-diol dehydrogenase (Q9TV69) (the identifiers refer to UniProt, the Universe protein resource, available at http://www.uniprot.org/). The search, conducted in October 2013, returned 2454 unique sequences. To remove redundancy among the retrieved sequences, they were clustered using BLASTclust (ftp://ftp.ncbi.nih.gov/blast/ documents/blastclust.html) to groups that are $80 \%$ identical along $70 \%$ of the sequence length. After clustering, 424 sequences remained. The query sequences were added and the set of 437 sequences were aligned using CLUSTALW (www.ebi.ac.uk/clustalw/). Non-full length sequences were removed, and the multiple sequence alignment of the remaining 426 sequences was used as an input to phylogenetic tree reconstruction using the Geneious Tree Builder with default parameters and with resampling (consensus of 100 trees). The tree is visualized in Fig. 1.

\section{Analytical procedures}

The extracellular compounds D-xylonate, xylitol, ethanol, glycerol, acetate, D-glucose and D-xylose were analysed by HPLC on a fast acid analysis column linked to an Aminex HPX-87H column (BioRad Labs, USA) with $2.5 \mathrm{mM}$ $\mathrm{H}_{2} \mathrm{SO}_{4}$ as eluent and a flow rate of $0.5 \mathrm{ml} \mathrm{min}^{-1}$. The column was maintained at $55^{\circ} \mathrm{C}$. Peaks were detected using a Waters 410 differential refractometer and a Waters 2487 dual wavelength UV (210 nm) detector. When D-xylonate was present, D-xylose concentrations were estimated by subtraction of the D-xylonate concentrations (detected by UV) from the combined D-xylose and D-xylonate peak detected by RI. Extracellular D-xylonate concentrations were also measured using the hydroxymate method (Lien 1959) as described by Toivari et al. (2010). Capillary electrophoresis as described in (Rovio et al. 2010; Turkia et al. 2013) was used for measuring D-gluconate concentrations in enzyme assays. Xylitol or Dsorbitol, from the enzymatic reduction reactions with Dxylose or D-fructose, respectively, were measured with a Dsorbitol/xylitol kit (Megazyme, Ireland) using a Konelab 20X automated analyser (Thermo Scientific, MA).

\section{Measurement of enzyme activity}

Enzyme activities were measured from cell extracts. Cell extracts were prepared with acid-washed glass beads in 2-ml tubes with screw-on caps with a roughly $1: 1: 1$ ratio of glass beads, cell pellet (wet) and lysis buffer $(50 \mathrm{mM}$ sodium phosphate buffer $\mathrm{pH}$ 6.8, $1 \mathrm{X}$ cOmplete EDTA-Free Protease 
 \\ 1.1.1.48, 1.1.1.46}

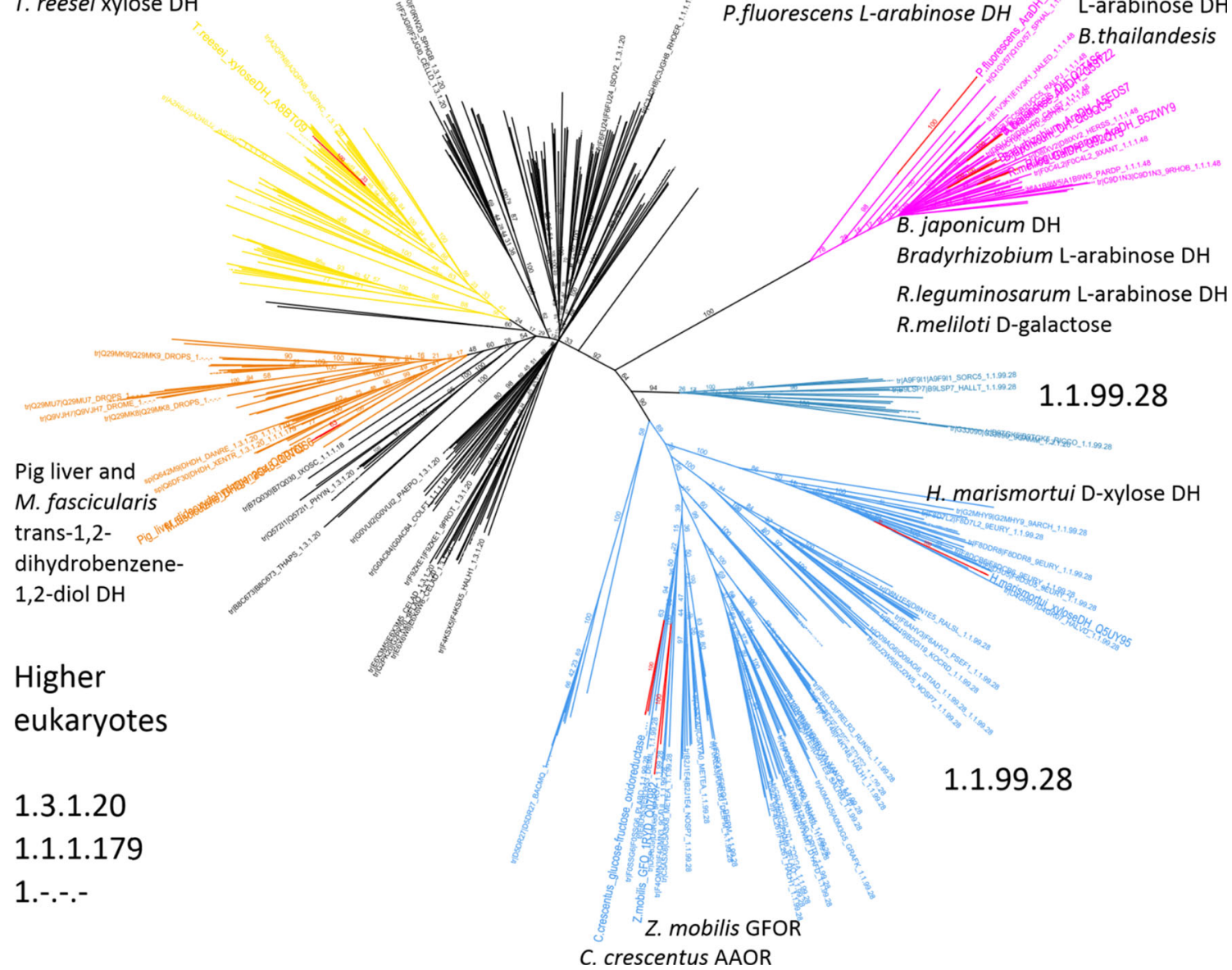

Fig. 1 A phylogenetic tree of aldose dehydrogenase enzymes of the Gfo/ $\mathrm{Idh} / \mathrm{MocA}$ family. The pink branch contains L-arabinose and D-galactose dehydrogenases. The light blue branch contains bacterial and archaeal GFOR, $C c$ AAOR and archaeal D-xylose dehydrogenases. The orange branch contains trans-1,2-dihydrobencene-1,2-diol dehydrogenases and

D-xylose dehydrogenases from higher eukaryotes. The yellow branch contains fungal sequences, including the $T$. reesei $\mathrm{D}$-xylose dehydrogenase. Sequence names are only shown for sequences with verified or tentative E.C. number annotation in the UniProt database

Inhibitor, Roche). The protein concentration of cell extracts was determined using the Bio-Rad Protein Assay kit, based on Bradford 1976 (Bradford 1976), with IgG as a standard.

D-Xylose dehydrogenase activity, with either $\mathrm{NAD}^{+}$or $\mathrm{NADP}^{+}$as a cofactor and $100 \mathrm{mM}$ D-xylose as substrate, was measured as described by Berghäll et al. (2007). For the measurement of glucose-fructose oxidoreductase activity, Dglucose $(400 \mathrm{mM})$ and D-fructose $(800 \mathrm{mM})$ were used as cosubstrates (comparable to concentrations in Zachariou and Scopes 1986). Alternatively D-xylose $(10 \mathrm{mM})$ alone was used as substrate. All reactions ( $400 \mu \mathrm{l}$ total volume) were carried out at $22{ }^{\circ} \mathrm{C}$ in $100 \mathrm{mM}$ potassium phosphate buffer $\mathrm{pH}$ 6.5. The reactions with $400 \mathrm{mM}$ D-glucose and $800 \mathrm{mM}$ $\mathrm{D}$-fructose were sampled at $0,15,30$ and $60 \mathrm{~min}$; reactions with $10 \mathrm{mM}$ D-xylose were sampled at $0,1,2$, and $3 \mathrm{~h}$. Reactions were stopped by heating at $100{ }^{\circ} \mathrm{C}$ for $10 \mathrm{~min}$. Xylitol, D-sorbitol or D-gluconate was measured as conversion products as described above. Activity was calculated as micromoles per minute product produced (per g of protein in cell extract), based on the concentration of D-sorbitol, xylitol or D-gluconate produced (depending on the substrate(s)).

\section{Results}

\section{Sequence similarities and genomic features of the $C$. crescentus aldose-aldose oxidoreductase}

The predicted amino acid sequence of the open reading frame from $C$. crescentus $\mathrm{CB} 15$ with locus tag $\mathrm{CC} 1225$, hereafter called aldose-aldose oxidoreductase, $C c$ AAOR, has $47 \%$ amino acid sequence identity with the glucose-fructose oxidoreductase from $Z$. mobilis ( $\mathrm{Zm}$ GFOR). It is annotated as a putative glucose-fructose oxidoreductase or NAD(P)-dependent oxidoreductase belonging to the $\mathrm{Gfo} / \mathrm{Idh} / \mathrm{MocA}$ family (PFAM family domains: PF01408, N-terminal and PF02894, 
C-terminal), members of which typically have a NAD(P)binding Rossmann fold. This family also contains L-arabinose/D-galactose dehydrogenases, D-xylose dehydrogenases and mammalian trans-1,2-dihydrobenzene-1,2-diol dehydrogenases with D-xylose dehydrogenase activity (Fig. 1). The D-xylose dehydrogenase xylB of $C$. crescentus, cloned and characterized earlier (Stephens et al. 2007; Toivari et al. 2012a, b), belongs to the family of short chain dehydrogenases (SDR) and shares little sequence similarity with enzymes of the Gfo/Idh/MocA family.

The ORF CC1225 corresponds to 366 predicted amino acids, including a putative periplasmic targeting signal sequence of 27 amino acids which was identified with the SignalP programme (www.cbs.dtu.dk/services/SignalP (Petersen et al. 2011)). The signal sequence region is shorter than that of the Z. mobilis GFOR, which consists of 52 amino acids. Sequences similar to the protein potentially coded by CC1225 of C. crescentus CB15 are also found in genomes of C. crescentus NA1000 (100\% identity) and Caulobacter segnis ATCC 21756 (85\% identity) (NCBI BLASTP 2.2.29 (Altschul et al. 1997)). Interestingly, the annotated sequence from C. crescentus NA1000 has an additional sequence of 32 amino acids at its $\mathrm{N}$-terminus, rendering it more similar in length to the signal sequence of $Z$. mobilis than the sequence from $C$. crescentus CB15. A DNA sequence coding for a similar region of amino acids is also found upstream of the predicted start codon of the CC1225 ORF in the CB15 strain, and in $C$. segnis ATCC 21756, suggesting the possibility that the translation start codon is not correctly predicted in the ORFs of $C$. segnis and C. crescentus CB15. The ORF CC1225 appears to be part of a putative operon of three genes in the genome of C. crescentus $\mathrm{CB} 15$, including the predicted genes encoding gluconolactonase (CC1224), glucose-fructose oxidoreductase (CC1225) and a protein with unknown function (CC1226).

\section{Expression and enzymatic activity of the putative oxidoreductase AAOR of $C$. crescentus and GFOR of $Z$. mobilis in $S$. cerevisiae}

The aaor (the CC1225 open reading frame) from the C. crescentus CB15 genome was codon optimized for $S$. cerevisiae and cloned under the $P G K 1$ promoter in a multicopy vector. Additionally, $C c$ aaor without the predicted 27 amino acids of the signal sequence was cloned. The $g$ for gene of Z. mobilis was cloned to the same vector backbone with and without its signal sequence. The plasmids were introduced into the $S$. cerevisiae lab strain CEN.PK 113-17A or alternatively into its derivative, deficient in the aldose reductase Gre3p (Table 1).

No D-xylose dehydrogenase activity was detected in the cell extracts of $S$. cerevisiae strains expressing either $C c$ aaor or Zm gfor using the described D-xylose dehydrogenase assay
(Berghäll et al. 2007), which measures reduction of NAD(P) ${ }^{+}$. However, cell extracts of strains expressing either the gfor of $Z$. mobilis or the aaor of $C$. crescentus showed production of D-sorbitol and D-gluconic acid in the glucose-fructose oxidoreductase activity assay (Table 2). The activity of $Z m$ GFOR in the cell extract from strain VTT-C-15933 was 120 or 40 fold higher than that of $C c$ AAOR in the cell extract from strain VTT-C-15928, when measured as D-sorbitol or D-gluconate, respectively (Table 2). Although $C c$ AAOR activity was clearly lower than $Z m$ GFOR activity, it was still higher than the background activity of the control strain, which showed no sorbitol and a 11 fold lower amount of D-gluconate production compared to the $C c$ aaor expressing cell extract. To test the activity with substrate concentrations that are more likely to be found inside the cell, a concentration of $10 \mathrm{mM}$ was selected for measuring the activity with D-xylose. Conversion of D-xylose as a single substrate at the chosen concentration was not detectable with cell extracts containing $Z m$ GFOR, whereas cell extracts with $C c$ AAOR showed 0.12-0.20 $\mu \mathrm{mol} \mathrm{min}^{-1} \mathrm{mg}$ protein $^{-1}$ activity, when measuring xylitol as the product (Table 2).

\section{Co-production of D-xylonate and xylitol from D-xylose with $S$. cerevisiae strains expressing $C c$ aaor}

S. cerevisiae strains expressing $C c$ aaor or $Z m$ gfor were grown in flasks in SC-ura medium containing $10 \mathrm{~g}$ Dglucose $\mathrm{l}^{-1}, 20 \mathrm{~g}$ D-xylose $\mathrm{l}^{-1}$ and $10 \mathrm{~g} \mathrm{CaCO}_{3} \mathrm{l}^{-1}$. The strain expressing $C$ c aaor produced $12.5 \mathrm{~g}$ D-xylonate $\mathrm{l}^{-1}$ and $11.5 \mathrm{~g}$ xylitol $1^{-1}$, whereas the strain expressing $Z m$ gfor and the control strain both produced $5 \mathrm{~g}$ xylitol $\mathrm{l}^{-1}$ but no Dxylonate $\left(<1 \mathrm{~g} \mathrm{l}^{-1}\right)$ (Fig. 2).

To assess whether the signal sequence present in the originally cloned $C c$ aaor and Zm gfor genes affected D-xylonate or xylitol production, yeast strains expressing $C c$ aaor and $Z m$ gfor versions without the signal sequences were also grown in flasks buffered with $\mathrm{CaCO}_{3}$. Again, the strain expressing $\mathrm{Cc}$ aaor (VTT-C-15935) produced $12 \mathrm{~g}$ D-xylonate $\mathrm{I}^{-1}$ and $11 \mathrm{~g}$ xylitol $\mathrm{l}^{-1}$ from $26 \mathrm{~g}$ D-xylose $\mathrm{l}^{-1}$ in $140 \mathrm{~h}$, whereas the strain expressing Z. mobilis gfor produced only xylitol, $5 \mathrm{~g} \mathrm{l}^{-1}$ (Fig. 2).

\section{Co-production of xylitol and D-xylonate with $S$. cerevisiae strains expressing aaor of $C$. crescentus in bioreactor cultures}

When strains expressing $C c$ aaor (with or without the native aldose reductase encoding gene GRE3) were grown in bioreactor culture, $10.8 \pm 0.8 \mathrm{~g}$ D-xylonate $\mathrm{l}^{-1}$ and $9.7 \pm 0.5 \mathrm{~g}$ xylitol $1^{-1}$ were produced from 20 to $25 \mathrm{~g}$ D-xylose $1^{-1}$ (Fig. 3). The molar ratio of D-xylonate and xylitol in the culture supernatant was $1.0 \pm 0.0$ throughout the cultivation. 
Table 2 Oxidoreductase activity in $S$. cerevisiae strains expressing $C c$ aaor or Zm gfor. Conversion of D-glucose $+\mathrm{D}$-fructose or D-xylose alone in crude cell extracts was measured with different substrate concentrations with either D-sorbitol and D-gluconate as products (D- glucose + D-fructose as substrates) or xylitol as a product of D-xylose

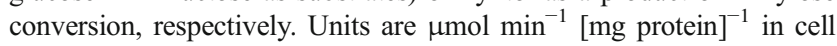
extract

\begin{tabular}{lllll}
\hline Strain & H-number & Glu $(400 \mathrm{mM})$ & Glu $(400 \mathrm{mM})$ & $\begin{array}{l}\text { Xyl }(10 \mathrm{mM}) \\
\text { Fru }(800 \mathrm{mM}) \\
\text { D-Gluconate }\end{array}$ \\
\hline Cc aaor & & $\begin{array}{l}\text { Fru }(800 \mathrm{mM}) \\
\text { D-Sorbitol }\end{array}$ & $1.40 \pm 0.27$ & $0.12 \pm 0.00$ \\
Cc aaor $\Delta$ GRE3 & VTT-C-15928 & $0.66 \pm 0.01$ & $0.86 \pm 0.74$ & $0.20 \pm 0.01$ \\
Zm gfor & VTT-C-15932 & $0.54 \pm 0.19$ & $49 \pm 7.7$ & $0.01 \pm 0.00$ \\
Control & VTT-C-15933 & $72 \pm 2.5$ & $0.13 \pm 0.01$ & $0.00 \pm 0.00$ \\
\hline
\end{tabular}

Strains expressing $C$ c aaor produced twice as much xylitol as the control strain (with GRE3) throughout the first $90 \mathrm{~h}$ cultivation, and still had $80 \%$ more xylitol than the control after 100 h. Deletion of GRE3 did not affect xylitol production when $C c$ aaor was expressed, but reduced xylitol production in its absence (Fig. 3). The Cc aaor strains produced Dxylonate and xylitol at volumetric rates of $108 \mathrm{mg}$ Dxylonate $\mathrm{l}^{-1} \mathrm{~h}^{-1}$ and $88 \mathrm{mg}$ xylitol $\mathrm{l}^{-1} \mathrm{~h}^{-1}$. The D-xylose uptake rate was $192 \mathrm{mg} \mathrm{D}$-xylose $\mathrm{l}^{-1} \mathrm{~h}^{-1}$, which was 2.8 times faster than the parent strain without $C c$ aaor but with an intact $G R E 3$ gene. Neither expression of $C c$ aaor nor deletion of $G R E 3$ affected biomass production in $S$. cerevisiae under these conditions (Fig. 3).

Both D-xylonate $\left(53 \pm 6 \mathrm{mg} \mathrm{g}^{-1}\right)$ and xylitol $\left(54 \pm 12 \mathrm{mg} \mathrm{g}^{-1}\right)$ accumulated intracellularly in $S$. cerevisiae expressing Cc aaor. The intracellular D-xylonate concentration was higher in the $\triangle G R E 3 C c$ aaor strain than in the strain with a functional Gre3p $\left(87 \pm 6 \mathrm{mg} \mathrm{g}^{-1}\right)$ but the intracellular xylitol concentration was similar $\left(50 \pm 4 \mathrm{mg} \mathrm{g}^{-1}, p>0.05\right)$. The control strain accumulated $41 \pm 4 \mathrm{mg} \mathrm{g}^{-1}$ xylitol, similar $(p>0.05)$ to that observed in the $C c$ aaor expressing strains, whereas deletion of GRE3 reduced this significantly $(p<0.05)$ to $25 \pm 1 \mathrm{mg} \mathrm{g}^{-1}$.

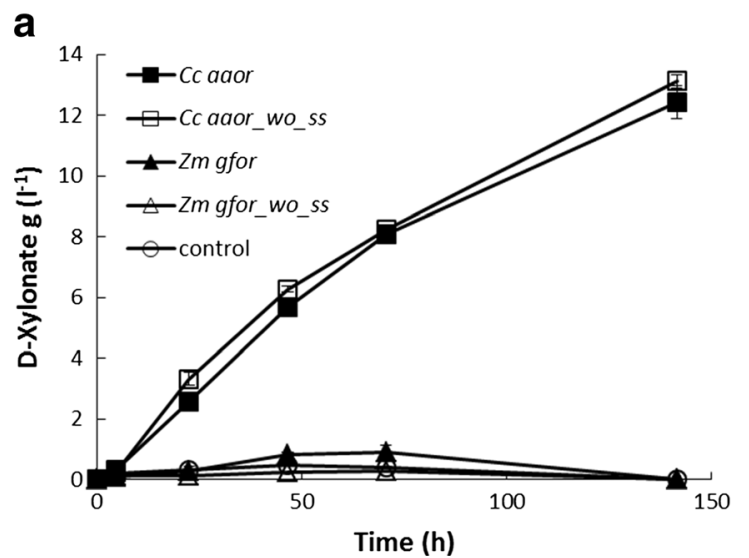

Co-expression of the D-xylonolactone lactonase encoding gene $x y l C$ from $C$. crescentus with the $C c$ aaor gene resulted in slightly increased production of both D-xylonate $\left(15 \mathrm{~g} \mathrm{l}^{-1}\right)$ and xylitol (11 $\mathrm{g} \mathrm{l}^{-1}$, Fig. 3), at initial volumetric rates of 140 and $130 \mathrm{mg} \mathrm{l}^{-1} \mathrm{~h}^{-1}$, respectively. Intracellular concentrations of D-xylonate $\left(63 \pm 7 \mathrm{mg} \mathrm{g}^{-1}\right)$ and xylitol $\left(43 \pm 4 \mathrm{mg} \mathrm{g}^{-1}\right)$ were similar to those observed in the strains lacking the lactonase.

\section{Discussion}

The newly identified enzyme (CC1225, named $C c$ AAOR) from $C$. crescentus was shown to convert in vitro the single aldose sugar D-xylose to D-xylitol, without an added cofactor. When the Cc aaor gene was expressed in S. cerevisiae, Dxylonate and xylitol were produced from D-xylose in equimolar concentrations. These results suggest that $C c$ AAOR functions as an aldose-aldose oxidoreductase (Fig. 4), being able to both reduce and oxidize D-xylose as a single substrate. The GFOR from $Z$. mobilis that belongs to the same protein family, does not need an added cofactor, but requires two substrates, preferably an aldose (D-glucose) and a ketose (D-fructose)

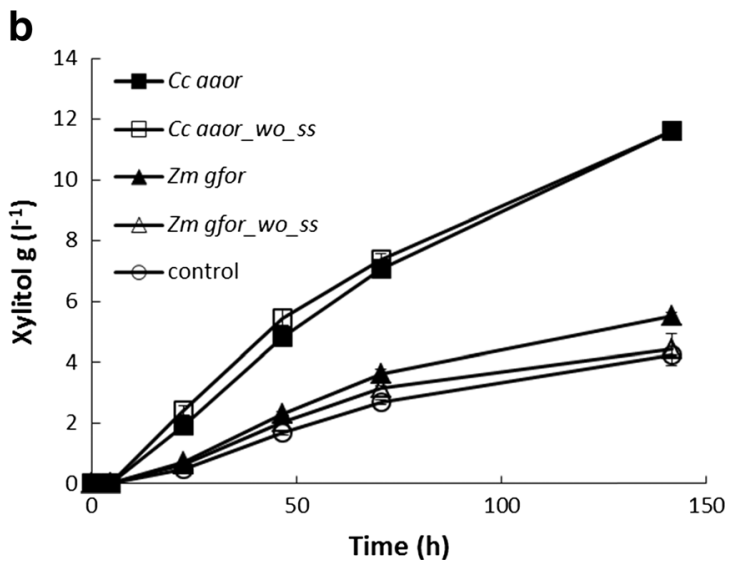

Fig. 2 Production of D-xylonate and xylitol in flask cultures of $S$. cerevisiae strains expressing aaor of C. crescentus or gfor of Z. mobilis. Medium containing D-glucose and D-xylose was buffered with $\mathrm{CaCO}_{3}$ 

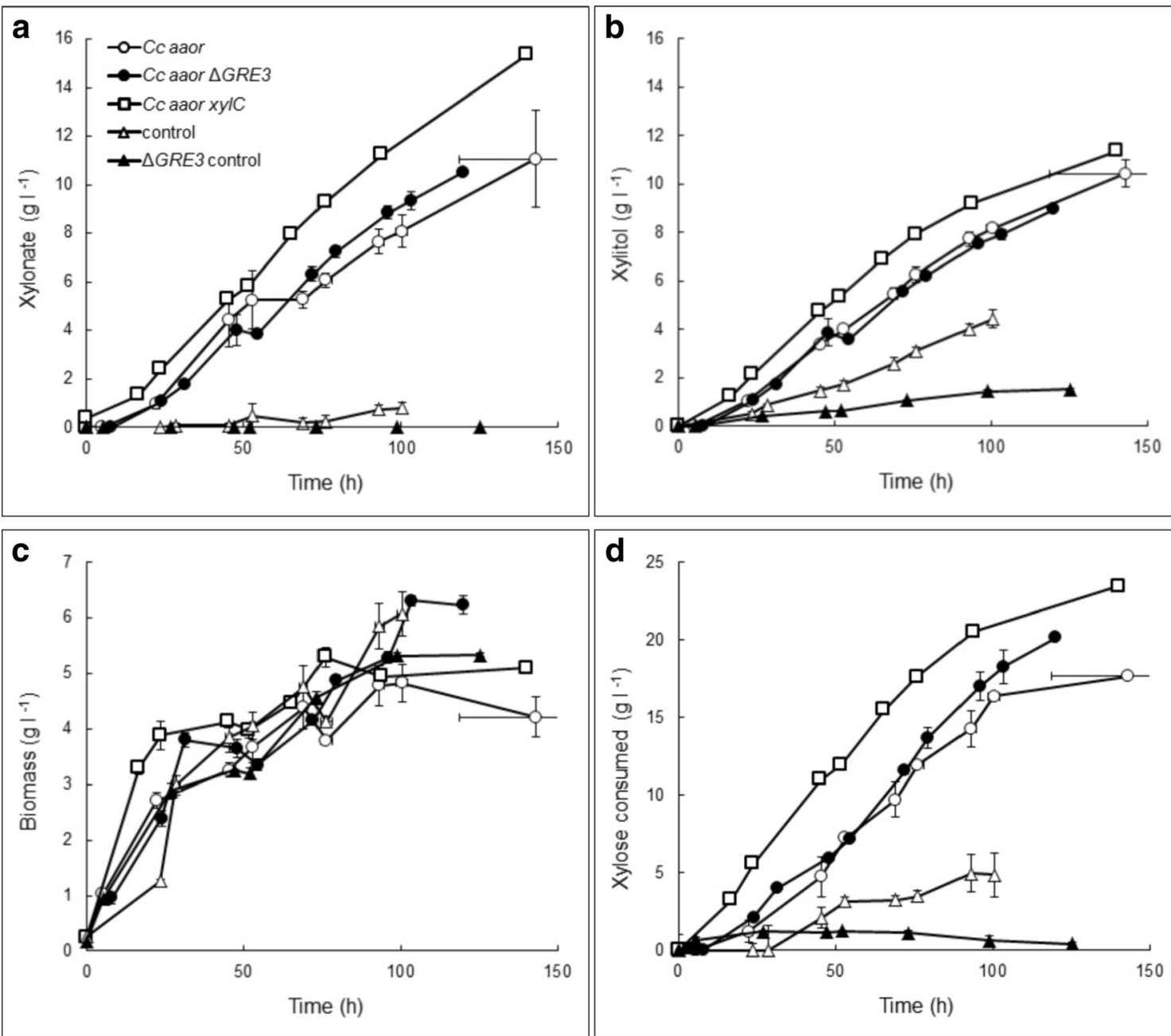

Fig. 3 Production of a D-xylonate, b xylitol, $\mathbf{c}$ biomass and $\mathbf{d}$ consumption of D-xylose by $S$. cerevisiae control (triangles) and strains expressing Cc aaor (circle and squares) in wild type (open symbols) or

$\Delta$ gre3 (solid symbols) strains. Cells were grown in bioreactors with D-

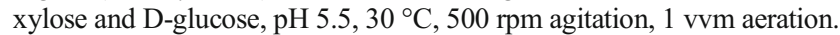
Error bars show SEM $(n=2-6)$

sugar. The difference between these two oxidoreductases was clear when they were expressed in $S$. cerevisiae and cultured in the presence of D-xylose: the $Z m$ GFOR containing strain did not produce D-xylonate from D-xylose and only similar concentrations of xylitol as the parental strain. To the best of our knowledge, $C c$ AAOR is the first single substrate aldosealdose reductase to be described.

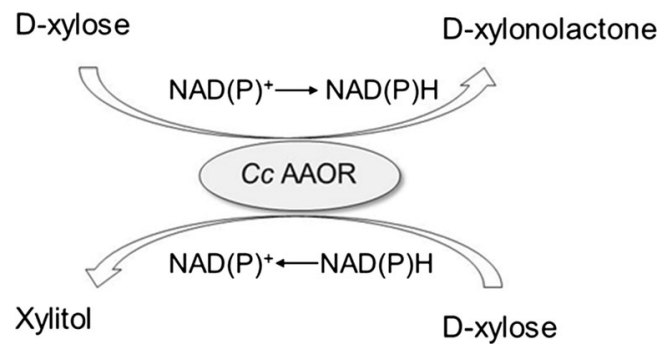

Fig. 4 Suggested oxidation-reduction reactions on D-xylose, carried out by the $C c$ AAOR enzyme from $C$. crescentus

Z. mobilis GFOR has been intensively studied and it is so far the only confirmed glucose-fructose oxidoreductase. It does not have very close homologues based on sequence similarity: the closest homologues are $53 \%$ identical, while $\mathrm{Zm}$ GFOR and $C c$ AAOR share $47 \%$ sequence similarity. $C c$ AAOR has higher sequence similarity to other putative enzymes (max. $65 \%$ ), which may also be interesting aldosealdose oxidoreductases.

Both $C c$ AAOR and $Z m$ GFOR possess a signal sequence of the TAT-pathway (twin-arginine translocation pathway). It is known that $Z m$ GFOR is folded as a pre-protein containing the signal sequence and is active both with and without it (Loos et al. 1993). The signal sequence for $C c$ AAOR (derived from $C$. crescentus $\mathrm{CB} 15$ ) is shorter than that of $Z m$ GFOR, and $C c$ AAOR was shown to be similarly active both with and without the signal sequence, when expressed in $S$. cerevisiae. Based on comparison of genome sequences from related bacterial species, it is possible that the native Cc AAOR from $C$. crescentus CB15 strain or from other 
Caulobacter species have longer signal sequences. With $Z m$ GFOR the signal sequence is cleaved during the translocation to the periplasmic space. We anticipate that the TAT-pathway is not functional in yeast and that the enzymes are located in the cytosol when expressed in S. cerevisiae. The physiological function of AAOR in $C$. crescentus remains currently unclear.

The $Z m$ GFOR enzyme has high $K_{\mathrm{m}}$ values, and high substrate concentrations ( $400 \mathrm{mM}$ to $1 \mathrm{M}$ ) have been used to measure its activity (Zachariou and Scopes 1986; Zhang et al. 2009). The Z. mobilis GFOR is located in the periplasmic space where the high concentrations needed may occur. In this study, the Zm gfor and $C c$ aaor genes were expressed intracellularly and thus, lower substrate concentrations were also tested with the single substrate $\mathrm{D}$-xylose. When high concentrations of D-glucose and D-fructose were used as substrates, both $Z m$ GFOR and $C c$ AAOR containing cell extracts showed activity in vitro, i.e. production of D-gluconic acid and $\mathrm{D}$-sorbitol via the oxidation-reduction reaction cycle, respectively. However, the activity of $C c$ AAOR was more than 100 -fold lower compared to the activity of $Z m$ GFOR. Interestingly, when a single substrate, D-xylose, was used in assessing the enzyme activities, $C c$ AAOR had 10-fold higher activity compared to $Z m$ GFOR. During the 3-h incubation an average of 180 and $17 \mathrm{mg} \mathrm{xylitol}^{-1}$ was formed by $C c$ AAOR and $Z m$ GFOR, respectively. It is possible that the $C c$ AAOR enzyme is inhibited by high substrate concentrations and/or that it reacts only with D-glucose (analogous to D-xylose) in the glucose-fructose oxidoreductase assay. D-Xylonate was not detected in the CE measurements performed, possibly because the system does not detected lactone forms and no lactonase enzyme was present. Measurements with optimized reaction conditions, different substrates and substrate concentrations, and more sensitive analytical methods are needed to further define the activity of this enzyme.

Despite the low $C c$ AAOR activity on D-xylose in vitro, a reasonable production rate of $\mathrm{D}$-xylonate and xylitol from D-xylose were obtained when $C c$ aaor was expressed in S. cerevisiae. D-Xylonate and xylitol were formed in equimolar concentrations, further supporting a mechanism of a single substrate aldose-aldose oxidoreduction for $C c$ AAOR (Fig. 4). There was no evidence of D-gluconate being produced from the D-glucose provided for growth, indicating that glycolysis efficiently competed with $C c$ AAOR for this hexose sugar. $S$. cerevisiae has several aldose reductases which result in production of xylitol from D-xylose. When $C c$ aaor was expressed in a $S$. cerevisiae strain deficient in the major aldose reductase Gre3p, again equal concentrations of D-xylonate and xylitol were formed, whereas without $C c$ aaor expression xylitol production was reduced by $\sim 70 \%$, further supporting the suggested mechanism of action of $C c$ AAOR. It seems that $C c$ AAOR can efficiently compete with Gre3p for the in vivo conversion of D-xylose.
The volumetric production rates and concentrations of Dxylonate obtained with $S$. cerevisiae expressing $C c$ aaor were much higher than those of D-xylonate-producing yeast strains expressing $\mathrm{NADP}^{+}$-dependent $\mathrm{D}$-xylose dehydrogenases of the Gfo/Idh/MocA family (xyd1 of T. reesei or SUS2DD from pig liver, Toivari et al. 2012a, b). Compared to the rate of Dxylonate production obtained with the strain expressing the NAD-dependent $C$. crescentus D-xylose dehydrogenase $x y l B$ of the short-chain dehydrogenases family in GRE3-deficient strain, the D-xylonate production rate of the $C c$ aaor strain was about half $\left(0.11\right.$ compared to $\left.0.23 \mathrm{~g} \mathrm{l}^{-1} \mathrm{~h}^{-1}\right)$, reflecting the co-production of xylitol with the $\mathrm{D}$-xylonate. The xylose consumption rates of $C c$ aaor or $x y l B$ expressing strains were comparable (0.19 and $0.21 \mathrm{~g} \mathrm{l}^{-1} \mathrm{~h}^{-1}$, respectively). With $C c$ aaor expressing strains the xylitol production rate and final titre were much higher compared to any of the D-xylose dehydrogenase expressing strains. As all D-xylose provided was converted to the two products D-xylonate and xylitol, the total yield of products on D-xylose was 1 .

The future bioeconomy needs novel solutions for production of chemicals in a sustainable way. New enzymes and engineered microbial strains enable development of versatile biorefinery concepts. The biosphere contains an enormous reserve of enzymes, of which we currently only apply a few. The present paper describes a novel aldose-aldose oxidoreductase, which is assumed to contain a bound cofactor and thus provides a self-sustained oxidation-reduction catalyst, able to produce both an oxidized and reduced product from a single substrate. Such enzymes provide interesting options for both in vitro and in vivo applications. The $C c$ AAOR enzyme was successfully used for conversion of the abundant, but today still inefficiently exploited biomass sugar D-xylose to two products, $\mathrm{D}$-xylonate and xylitol, in a single reaction by a single yeast strain. With increasing xylitol demand and potential interest in various applications of D-xylonate this enzyme provides an interesting option for their co-production. The differences between the $C c$ AAOR and the $Z m$ GFOR are yet to be determined in detail, but the differences in action are likely due to structural differences, particularly in the Nterminal region. We are currently in the process of characterizing the properties and determining the crystal structure of C. crescentus AAOR to obtain a more thorough understanding of the enzyme and to be able to fully exploit its potential.

Acknowledgments Technical assistance of Tarja Laakso and Outi Könönen is gratefully acknowledged. Stella Rovio and Heidi Turkia are acknowledged for the CE-analytics. The financial support by the Academy of Finland through the Centre of Excellence in White Biotechnology-Green Chemistry (grant 118573), and by the European Commission through the Sixth Framework Programme Integrated Project BioSynergy (038994-SES6) is acknowledged. Financial support from the VTT Graduate School is acknowledged (Yvonne Nygård).

Conflict of interest The authors declare that they have competing interests. 
Open Access This article is distributed under the terms of the Creative Commons Attribution 4.0 International License (http:// creativecommons.org/licenses/by/4.0/), which permits unrestricted use, distribution, and reproduction in any medium, provided you give appropriate credit to the original author(s) and the source, provide a link to the Creative Commons license, and indicate if changes were made.

\section{References}

Altschul SF, Madden TL, Schaffer AA, Zhang J, Zhang Z, Miller W, Lipman DJ (1997) Gapped BLAST and PSI-BLAST: a new generation of protein database search programs. Nucleic Acids Res 25: 3389-3402

Berghäll S, Hilditch S, Penttilä M, Richard P (2007) Identification in the mould Hypocrea jecorina of a gene encoding an NADP(+): dxylose dehydrogenase. FEMS Microbiol Lett 277:249-253

Bradford MM (1976) A rapid and sensitive method for the quantitation of microgram quantities of protein utilizing the principle of protein-dye binding. Anal Biochem 72:248-254

Cao Y, Xian M, Zou H, Zhang H. Metabolic engineering of Escherichia coli for the production of xylonate. PLoS One. 2013;8(7):e67305.

Chun BW, Dair B, Macuch PJ, Wiebe D, Porteneuve C, Jeknavorian A (2006) The development of cement and concrete additive: based on xylonic acid derived via bioconversion of xylose. Appl Biochem Biotechnol 129-132:645-658

Dumon C, Song L, Bozonnet S, Faure R, O'Donohue MJ (2012) Progress and future prospects for pentose-specific biocatalysts in biorefining. Process Biochem 47:346-357

Entian KD, Kötter P (1998) Yeast mutant and plasmid collections In: Brown AJP, Tuite MF, editors. Yeast gene analysis. Method Microbiol 26:431-449

Hallborn J, Walfridsson M, Airaksinen U, Ojamo H, Hahn-Hägerdal B, Penttilä M, Keränen S (1991) Xylitol production by recombinant Saccharomyces cerevisiae. Biotechnology (N Y) 9:1090-1095

Lien OG (1959) Determination of gluconolactone, galactonolactone and their free acids by the hydroxamate method. Anal Chem 31:1363-1366

Liu H, Valdehuesa KN, Nisola GM, Ramos KR, Chung WJ (2012) High yield production of $\mathrm{D}$-xylonic acid from $\mathrm{D}$-xylose using engineered Escherichia coli. Bioresour Technol 115:244-248

Loos H, Sahm H, Sprenger GA (1993) Glucose-fructose oxidoreductase, a periplasmic enzyme of Zymomonas mobilis, is active in its precursor form. FEMS Microbiol Lett 107:293-298

Millner OE, Clarke RP, Titus GR (1994) Clarifiers for polyolefins and polyolefin compositions containing same
Nidetzky B, Neuhauser W, Haltrich D, Kulbe KD (1996) Continuous enzymatic production of xylitol with simultaneous coenzyme regeneration in a charged membrane reactor. Biotechnol Bioeng 52:387-396

Nygård Y, Toivari MH, Penttilä M, Ruohonen L, Wiebe MG (2011) Bioconversion of D-xylose to D-xylonate with Kluyveromyces lactis. Metab Eng 13:383-391

Peng F, Peng P, Xu F, Sun RC (2012) Fractional purification and bioconversion of hemicelluloses. Biotechnol Adv 30:879-903

Petersen TN, Brunak S, von Heijne G, Nielsen H (2011) SignalP 4.0: discriminating signal peptides from transmembrane regions. Nat Methods 8:785-786

Piersma SR, de Vries S, Duine JA (1997) Nicotinoprotein alcohol/ aldehyde oxidoreductases. Enzymes with bound NAD(P) as cofactor. Adv Exp Med Biol 414:425-434

Rovio S, Kalliola A, Sirén H, Tamminen T (2010) Determination of the carboxylic acids in acidic and basic process samples by capillary zone electrophoresis. J Chromatog A 1217:1407-1413

Stephens C, Christen B, Fuchs T, Sundaram V, Watanabe K, Jenal U (2007) Genetic analysis of a novel pathway for D-xylose metabolism in Caulobacter crescentus. J Bacteriol 189:2181-2185

Toivari M, Nygård Y, Kumpula EP, Vehkomäki ML, Bencina M, Valkonen M, Maaheimo H, Andberg M, Koivula A, Ruohonen L, Penttilä M, Wiebe MG (2012a) Metabolic engineering of Saccharomyces cerevisiae for bioconversion of D-xylose to Dxylonate. Metab Eng 14:427-436

Toivari M, Vehkomäki ML, Nygård Y, Penttilä M, Ruohonen L, Wiebe MG (2013) Low pH D-xylonate production with Pichia kudriavzevii. Bioresour Technol 133:555-562

Toivari MH, Nygård Y, Penttilä M, Ruohonen L, Wiebe MG (2012b) Microbial D-xylonate production. Appl Microbiol Biotechnol 96: $1-8$

Toivari MH, Ruohonen L, Richard P, Penttilä M, Wiebe MG (2010) Saccharomyces cerevisiae engineered to produce D-xylonate. Appl Microbiol Biotechnol 88:751-760

Turkia H, Holmström S, Paasikallio T, Sirén H, Penttilä M, Pitkänen JP (2013) Online capillary electrophoresis for monitoring carboxylic acid production by yeast during bioreactor cultivations. Anal Chem 85(20):9705-9712

Winkelhausen E, Kuzmanova S (1998) Microbial conversion of D-xylose to xylitol. J Ferment Bioeng 86:1-14

Zachariou M, Scopes RK (1986) Glucose-fructose oxidoreductase, a new enzyme isolated from Zymomonas mobilis that is responsible for sorbitol production. J Bacteriol 167:863-869

Zamora F, Bueno M, Molina I, Iribarren JI, Munoz-Guerra S, Galbis JA (2000) Stereoregular copolyamides derived from D-xylose and Larabinose. Macromolecules 33:2030-2038

Zhang X, Chen G, Liu W (2009) Reduction of xylose to xylitol catalyzed by glucose-fructose oxidoreductase from Zymomonas mobilis. FEMS Microbiol Lett 293:214-219 